\title{
SURVEYS IN THE ADC ARCHIVE
}

\author{
N.G. ROMAN \\ HughesSTX/ADC, Goddard Space Flight Center
}

\section{Introduction}

The ADC archive contains more than 800 catalogs and extensive tables from more than 750 journal articles. The archive is increasing steadily at the rate of about 20 data sets per month. This archive can be accessed either by anonymous FTP or by a World Wide Web (WWW) browser at

$$
\text { http://adc.gsfc.nasa.gov/ }
$$

\section{Searching the ADC Archive}

In addition to the archive, which is divided into separate subdirectories for catalogs and the journal files, both methods also access several other directories including address directories for both individuals and institutions and a small collection of software for catalog searching. At each level, there is a "key" which lists the content of the directory and its subdirectories (except for the individual catalog or article level). With FTP access, this key may be downloaded and searched for subjects and authors of interest.

The WWW access provides a full text search capability in the document which describes each file. On the WWW, the document can be reviewed to determine if the files are of interest. All files except the documents are stored in compressed ASCII form. They can be retrieved either as compressed or as uncompressed files or converted to FITS.

\section{Sample Search}

A search of the archives with the word "survey" returned a list of 338 items. The following are some examples: 
STARS (OPTICAL)

X RAY

EUVE

INFRARED

RADIO
Durchmusterungen

Michigan Spectral Classification Catalogs

Case Low Dispersion Survey

General Catalog of Variable Stars

Lowell, Lick, and Luyten Proper Motion Catalogs

Catalog of Radial Velocities

Luminous Stars in the Milky Way

Northern M Dwarfs

Thick Disk Chemical Abundance Distribution

GALAXIES (OPTICAL) Rich Clusters of Galaxies (Abell)

CfA Redshift Survey

Multicolor Survey of High Redshift Quasars

APM Bright Galaxy Catalog

Catalog of High-Redshift Quasars

Spectroscopic Survey of Faint QSO's

Pisces-Perseus Supercluster

Third Reference Catalog of Bright Galaxies

HEAO A-1 X-ray Source Catalog

ROSAT Wide Field Camera All-sky Survey

Wisconsin Soft X-ray Diffuse Background All-sky Survey

$\mathrm{X}$-ray Emission at the Low-Mass End

Einstein Data Bass of Quasars

X-ray AGN Content of the Molonglo $408 \mathrm{MHz}$ Survey

ROSAT All-Sky Bright Source Catalogue

First EUVE Source Catalog

Second Extreme Ultra-Violet Explorer Catalog

EUV Explorer Bright Source List

Far UV Point Source

IRAS Catalogs of Point Sources and Faint Sources

Revised AFGL Infrared Sky Survey and Supplement

Two-micron Sky Survey

IRAS Faint Source Catalog

IRAS Redshift Survey

Equatorial Infrared Catalog

K Survey of Ori-A Molecular Cloud

New Infrared Survey of Northern Planetaries

Catalogs from Deep $327 \mathrm{MHz}$ Westerbork Survey

Bell Laboratories HI Survey

Parkes-MIT-NRAO (PMN) Surveys

Shapley-Ames Catalog at $2.8 \mathrm{~cm}$

Fourth MIT-Green Bank $5 \mathrm{GHz}$ Survey

Molecular Outflow Sources

53,522 Sources at $4.58 \mathrm{GHz}$

$7 \mathrm{C}$ Survey of Radio Sources 